\title{
Modeling the distributional dynamics of attention and semantic interference in word production
}

\author{
Aitor San José $^{\mathrm{a}, \mathrm{b}, *}$, Ardi Roelofs ${ }^{\mathrm{c}}$, Antje S. Meyer ${ }^{\mathrm{a}, \mathrm{c}}$ \\ ${ }^{\text {a }}$ Max Planck Institute for Psycholinguistics, Nijmegen, the Netherlands \\ b International Max Planck Research School for Language Sciences, Nijmegen, the Netherlands \\ ${ }^{\mathrm{c}}$ Donders Institute for Brain, Cognition and Behaviour, Radboud University, Nijmegen, the Netherlands
}

\section{A R T I C L E I N F O}

\section{Keywords:}

Attention

Distributional analyses

Executive control

Picture-word interference

Reaction time

Word production

\begin{abstract}
A B S T R A C T
In recent years, it has become clear that attention plays an important role in spoken word production. Some of this evidence comes from distributional analyses of reaction time (RT) in regular picture naming and pictureword interference. Yet we lack a mechanistic account of how the properties of RT distributions come to reflect attentional processes and how these processes may in turn modulate the amount of conflict between lexical representations. Here, we present a computational account according to which attentional lapses allow for existing conflict to build up unsupervised on a subset of trials, thus modulating the shape of the resulting RT distribution. Our process model resolves discrepancies between outcomes of previous studies on semantic interference. Moreover, the model's predictions were confirmed in a new experiment where participants' motivation to remain attentive determined the size and distributional locus of semantic interference in picture naming. We conclude that process modeling of RT distributions importantly improves our understanding of the interplay between attention and conflict in word production. Our model thus provides a framework for interpreting distributional analyses of RT data in picture naming tasks.
\end{abstract}

\section{Introduction}

A workhorse in the study of spoken word production is the pictureword interference (PWI) paradigm (e.g., Damian \& Martin, 1999; Schriefers, Meyer, \& Levelt, 1990). In a typical PWI study, participants are instructed to name a picture (e.g., of a dog) while trying to ignore a written distractor word superimposed onto the picture. In studies of lexical selection, the distractor words are typically semantically related (e.g., fish) or unrelated (e.g., tree) to the picture. It is consistently found that, relative to unrelated distractors, related distractors yield a Strooplike increase of RT called semantic interference. Traditionally, this interference effect has been measured as the difference in mean RT between the two conditions. Recently, however, researchers have begun to explore the distributional dynamics of semantic interference using tools such as ex-Gaussian analyses, which model RT distributions as the convolution of a Gaussian and an exponential component (e.g., Balota, Yap, Cortese, \& Watson, 2008; Luce, 1986). The Gaussian component is described by the parameters $\mu$ and $\sigma$, which correspond to its mean and standard deviation. The $\tau$ parameter describes the exponential component (viz., its mean and standard deviation) and is therefore a measure of skewness. The mean of the distribution is the sum of $\mu$ and $\tau$. Thus, any mean RT effect can be decomposed into a $\mu$ effect (a shift of the distribution) and a $\tau$ effect (a difference in skewness).

PWI is a picture-word analog of the color-word Stroop task, which is a gold standard of attentional measures (MacLeod, 1991). Clearly, attention also plays a role in PWI, since interference from distractor words needs to be resolved (e.g., Kleinman, 2013; Piai, Roelofs, \& Schriefers, 2014; Roelofs, 2003; Schnur \& Martin, 2012). It is therefore possible that differences in results from studies using RT distributional analyses are due to differences in the deployment of attention. This points to the need for a computational account of how the properties of RT distributions come to reflect the effect of attentional processes and how these may modulate the amount of conflict between lexical representations. Yet we lack such a computational account. The aim of the present article is to fill this theoretical gap.

The fundamental hypothesis in the article is that lapses of attention play a key role in determining the shape of an RT distribution (cf. Jongman, Roelofs, \& Lewis, 2020; Jongman, Roelofs, \& Meyer, 2015). Nineteenth century pioneers of research on attention and performance, like James and Wundt, already assumed a fundamental influence of

\footnotetext{
* Corresponding author at: Max Planck Institute for Psycholinguistics, P. O. Box 310, 6500 AH Nijmegen, the Netherlands.

E-mail address: Aitor.SanJose@mpi.nl (A. San José).
} 
fluctuations of attention on performance. James (1890) stated: "There is no such thing as voluntary attention sustained for more than a few seconds at a time" (p. 420). In discussing fluctuations, Wundt (1908) argued that external and internal disturbances of attention ("äußere und innere Störungen der Aufmerksamkeit”, p. 581) may cause a frequency distribution to become more asymmetrical, for example, by increasing the slow tail. We refer to Woodworth (1938) and Woodworth and Schlosberg (1954) for reviews of the early literature.

Our article is structured as follows. In the remainder of this introduction section, we first discuss the empirical findings from PWI that motivated our work, and also briefly describe the WEAVER ++ model. This is a computational model of word planning and its attentional control (e.g., Roelofs, 2003), which accounts for RT findings. In Section 2 , we discuss the way the model was extended to account for attentional lapses. In Section 3, we report on simulations that tested the model's ability to quantitatively account for the difference in results between methodologically comparable PWI studies in the literature as arising solely from differences in the properties of lapses. In Sections 4 and 5, we further explore the interplay between lapses of attentional control and lexical competition in modulating the locus of the semantic interference effect in the RT distribution. In Section 6, we show that the model's predictions were confirmed in a new experiment where participants' motivation to remain attentive determined the size and distributional locus of semantic interference in PWI.

\subsection{Distributional findings on picture-word interference}

Scaltritti, Navarrete, and Peressotti (2015a, 2015b) used ex-Gaussian analyses to explore the distributional properties of the semantic interference effect in PWI. In two experiments, they found that the semantic effect was significantly present in $\tau$, although their first experiment also showed a marginally significant effect in $\mu$. They suggested that semantic interference might be selectively linked to the $\tau$ parameter. However, in a subsequent study by Roelofs and Piai (2017), the semantic interference effect yielded not only a significant increase in $\tau$ but also in $\mu$. This pattern was replicated in Roelofs and Piai's reanalysis of an earlier PWI study by Piai, Roelofs, and Van der Meij (2012). Finally, an earlier study by (Piai, Roelofs, \& Schriefers, 2011), Experiment 1) found an effect of semantic interference in $\mu$ alone. Taken together, these results suggest that semantic interference can cause not only an increase in skewness in the RT distribution, but also an overall distributional shift. The distributional shift is currently accounted for in WEAVER++ (Roelofs, 2008) as arising from increased competition between semantically related lemmas (Levelt, Roelofs, \& Meyer, 1999; Roelofs, 1992, 2018). In the present work, we examine whether the WEAVER++ model can also account for the abovementioned skewing effects as arising from attentional lapses.

\subsection{The WEAVER++ model}

WEAVER ++ is a symbolic computational model that was originally designed to explain mean RT effects from picture naming tasks (Roelofs, 1992) and was later extended to account for other task variants, such as the color-word Stroop task (e.g., Roelofs, 2003; Roelofs \& Hagoort, 2002), and for semantic interference in continuous and blocked-cyclic naming (Roelofs, 2018). WEAVER ++ consists of a declarative network and a procedural component. The declarative network is made up of three types of symbolic nodes, which are arranged into three strata: the concept, the lemma (syntactic), and the word-form stratum. When a concept node is activated (e.g., by a picture), activation spreads to the lemma it is linked to. Moreover, some of this activation spreads to semantically related lemmas, mediated by concept-to-concept links. A lemma is selected by means of a procedural rule (see below). Activation spreads further to the word-form stratum, which consists of morpheme, phoneme, and syllable program layers. Lexical access is complete when the correct syllable program nodes are selected, after which the only remaining process is articulation (not included in WEAVER ++ ). For a thorough description of WEAVER++'s architecture, the reader is referred to Levelt et al. (1999) and Roelofs (1992, 2003, 2008, 2018). Since the simulations in the present work are only concerned with semantic effects at the lemma level, only the processes up to lemma selection were modeled.

WEAVER++'s procedural component consists of a set of conditionaction rules which modulate the activation of nodes in the declarative network so as to achieve the selection of the target lemma. WEAVER++ uses the following production rules for picture naming and picture-word interference:

Production rule 1 IF the task goal is to name the picture AND $\operatorname{concept}(x)$ was evoked by the picture

THEN select $\operatorname{concept}(x)$ AND flag $\operatorname{concept}(x)$ as a goal AND enhance the activation of concept $(x)$.

Production rule 2 IF the activation of $\operatorname{lemma}(x) \geq$ selection_threshold AND lemma $(x)$ is linked to the goal concept

THEN select lemma $(x)$.

Production rule 3 IF the task goal is to name the picture AND input is received from a word

THEN block out the word input.

In WEAVER++, the semantic interference effect in the PWI paradigm arises as follows. When presented with a picture, the model selects the concept associated to it and enhances its activation according to Production rule 1 . The lemma corresponding to the written distractor word is blocked out according to Production rule 3 . The selection of the target lemma is then achieved through Production rule 2 by ensuring that the target lemma has achieved a high enough activation level relative to the activation of other lemmas in the network. The actual moment of selection is determined through an activation ratio, such that the selection of the target lemma will be delayed as a function of the activation received by its competitors. The semantic interference effect thus arises because semantically related distractors cause a delay in lemma selection, compared to unrelated distractors.

\subsection{The implementation of attentional lapses}

In our implementation, we assumed that lapses of goal maintenance reflect brief moments of disengagement from the task, due to either internal or external stimuli momentarily capturing the focus of attention (cf. Wundt, 1908). We assume that these lapses of goal maintenance cause WEAVER++'s top-down control to be momentarily interrupted, since its production rules are dependent on such task goal. We reasoned that the most likely mechanism to be affected by lapses is the top-down enhancement in Production rule 1, since this mechanism needs to be applied in a sustained fashion throughout the entire trial and plays an active and direct role in countering semantic interference. Accordingly, during a lapse of top-down enhancement, the declarative network runs unsupervised, and spreading activation allows for any existing semantic interference to quickly build up, thus delaying selection and increasing RT on affected trials. Technically, this implementation implies that goal maintenance in the model is isomorphic with the capacity to consistently apply top-down enhancement to target concepts (i.e., to apply Production rule 1). However, the model yields equivalent results when lapses affect other forms of top-down control (e.g., production rule 3). Thus, we can define goal maintenance in the model more broadly as the ability to consistently apply task-goal-directed executive control in a sustained and uninterrupted fashion (cf. Kane \& Engle, 2003). Because it is difficult to make a clear-cut distinction between lapses of goal maintenance and lapses of top-down control, we henceforth use the two terms interchangeably.

\section{Simulation methods}

In order to implement lapses, three new parameters were introduced in the model: lapse_rate, lapse_duration_mean and lapse_duration_stdev. The 
parameter lapse_rate determines the probability of a lapse occurring at any given time, provided that a lapse is not currently taking place. The duration of a lapse is then sampled from a Gaussian distribution with a mean of lapse_duration_mean and a standard deviation of lapse_duration_stdev. In the following sections, we show how the behavior of these three parameters determines whether semantic interference shows up in $\tau$, $\mu$, or both.

\subsection{Trial procedure}

Following Roelofs (1992), the language network consisted of six concepts and their corresponding lemmas. On each trial, the target lemma to be selected was dog. Semantically related trials were modeled by assuming the presence of the written distractor fish. On semantically unrelated trials, the distractor was tree. Picture-word interference was modeled by assuming that the target picture activates the concept associated with it, whereas a written distractor activates its corresponding lemma directly. Selection is then achieved through the procedural rules described above.

\subsection{Sampling procedure}

In previous versions of WEAVER ++ , RT distributions were computed using a mathematical formalism (e.g., Roelofs, 2008). For the present simulations, a sampling procedure was introduced, which makes the derivation of RT stochastic. Each sample was obtained by running an independent trial in the model. The RT value for each sample was the time point at which the target lemma was selected. WEAVER++'s step_size parameter, which determines how often the network's activation is evaluated and updated (see Roelofs, 1992), was set to $1 \mathrm{~ms}$ for all simulations, so as to obtain maximal accuracy in the sampling of RT. For the parameter optimization in Simulation 1, the number of samples per condition was allowed to increase gradually from 250 to 2500 , as a function the number of iterations. This was to speed up parameter exploration at the beginning of the optimization without compromising exploitation at the end. All the results reported throughout the article were obtained with a sample size of 50,000 per condition, so as to maximize convergence.

\subsection{Delta deciles}

Delta decile plots of the simulation results were computed as follows. First, all raw RT samples from each distractor condition were rank ordered and divided into 10 bins. Deciles were obtained by computing the mean RT of each bin. Delta deciles were then computed by subtracting the deciles in the unrelated condition from the deciles in the semantically related condition. Consequently, delta deciles index the mean size of the semantic interference effect for each decile.

\subsection{Delta ex-Gaussian analyses}

Ex-Gaussian analyses were carried out on the raw RT samples separately for each condition using the $\mathrm{R}$ function mexgauss from the retimes package (Massidda, 2013). As with delta deciles, delta exGaussian parameters were calculated by subtracting the parameters in the unrelated condition from the parameters in the semantically related condition. Thus, $\Delta \mu$ indexes the magnitude of distributional shifting resulting from semantic interference, while $\Delta \sigma$ indexes the corresponding change in standard deviation, and $\Delta \tau$ indexes the change in skewness.

\section{Simulation 1: Addressing empirical discrepancies}

\subsection{Aim}

The aim of the first simulation was to test the model's ability to account for the conflicting distributional findings of previous studies as arising from differences in attentional engagement. In particular, we simulated the data from the studies of Scaltritti et al. (2015a, b, Experiment 3) and Roelofs and Piai (2017). As discussed earlier, Scaltritti et al. found an effect of semantic interference in $\tau$ alone while Roelofs and Piai found it in both $\mu$ and $\tau$. These two studies were chosen for being the most methodologically similar ones, differing primarily in the stimuli, language, and number of stimulus repetitions. Since Scaltritti et al.'s first experiment found a marginally significant effect of semantic interference in $\mu$, we chose to simulate their third experiment instead, as its results contrast more sharply with those of Roelofs and Piai. In order to test the hypothesis that the distributional differences between the two chosen experiments can arise from group differences in the rate and duration of lapses, the model parameters were optimized to account for the two empirical data sets by differing primarily in the lapse parameters (i.e., lapse_rate, lapse_duration_mean, and lapse_duration_stdev). Details of the optimization procedure can be found in Appendix A.

\subsection{Results and discussion}

The lapse parameter values for each data set are given in Table 1, together with the resulting model error (i.e., root mean squared error, RMSE) and delta ex-Gaussian parameters. As shown by the table, RMSE was low for both data sets, indicating good model fit.

The delta decile plots in Fig. 1 show that the model was able to replicate the distributional patterns in both data sets. Specifically, the model correctly captures the fact that the semantic effect in Roelofs and Piai (2017) manifested itself as both an increase in skewness and an overall distributional shift and is therefore present in all the deciles. In contrast, in Scaltritti et al. (2015a, 2015b) Experiment 3, it was largely captured by an increase in skewness and is thus only salient from decile 5 onward. Note, however, that the semantic effect was not 0 for any decile, in line with the findings of Roelofs (2008) that the semantic effect is present throughout the entire distribution.

The results of the delta ex-Gaussian analyses converge with the decile plot results. As shown in Fig. 2, the model yields the same pattern as the data, with the semantic effect showing up in both $\mu$ and $\tau$ for the data of Roelofs and Piai (2017) but only in $\tau$ for the Scaltritti et al. (2015a, 2015b) data. As Table 1 shows, the model predicted the data of Roelofs and Piai as arising from lapses that were on average $66 \mathrm{~ms}$ shorter than for the Scaltritti et al. data. The lapse_duration_stdev value was, however, $95 \mathrm{~ms}$ larger in the Roelofs and Piai data than in the Scaltritti et al. data, indicating more variation in the duration of lapses. Finally, the rate of lapses was almost 0.026 higher in the Roelofs and Piai data, meaning that lapses were almost seven times as frequent in the Roelofs and Piai data compared to the Scaltritti et al. data. Thus, the difference in lapse parameter values between the two data sets did not follow a single direction. Proportionally, however, the difference in lapse_rate was much larger than the difference in the duration parameters. This suggests that the lapse_rate parameter can be considered the main driving force behind the distributional differences obtained in this simulation. Accordingly, since lapses had a greater impact on the semantic effect for Roelofs and Piai, we can infer that the model predicted these results as arising from worse goal maintenance for Roelofs and Piai than for Scaltritti et al.

However, it needs to be pointed out that the optimizer did not show signs of convergence indicating a very irregular parameter space and pointing to the existence of several parameter configurations that may in principle be able to explain the data similarly well. It is thus possible that the model could predict the Roelofs and Piai (2017) data as arising from better, rather than worse, maintenance than the Scaltritti et al. (2015a, 2015b) data. In any case, regardless of the directionality of goal maintenance differences, the results of Simulation 1 confirm the hypothesis that differences in fluctuations of top-down control are a plausible explanation for the distributional differences between the two studies. 
Table 1

Lapse parameters, delta ex-Gaussian parameters, and RMSE for each data set modeled in Simulation 1.

\begin{tabular}{|c|c|c|c|c|c|c|c|}
\hline Data set & lapse_rate & lapse_dur_mean & lapse_dur_stdev & $\Delta \mu$ & $\Delta \sigma$ & $\Delta \tau$ & RMSE \\
\hline Roelofs and Piai (2017) & 0.0302 & $118 \mathrm{~ms}$ & $120 \mathrm{~ms}$ & 22.4 & 11.5 & 15.33 & 3.46 \\
\hline Scaltritti et al. (2015 a, b, Exp 3) & 0.0044 & $184 \mathrm{~ms}$ & $25 \mathrm{~ms}$ & 3.5 & -1.98 & 16.32 & 4.66 \\
\hline
\end{tabular}
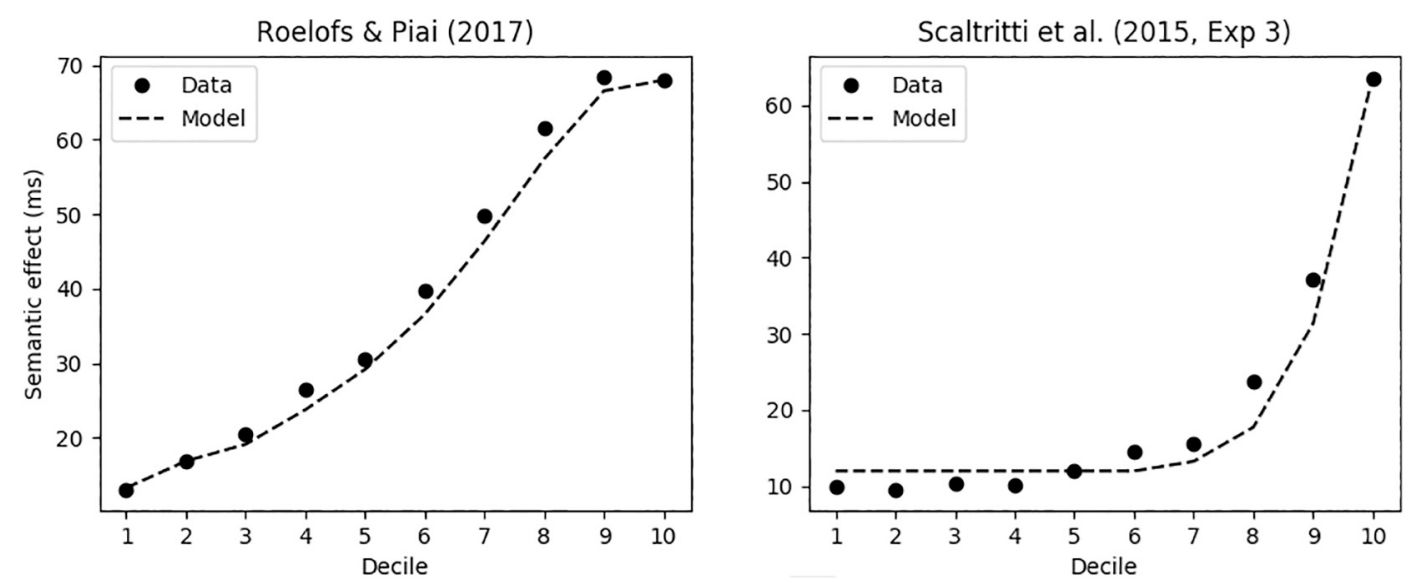

Fig. 1. Delta decile plots of the semantic effect in the model versus empirical data.
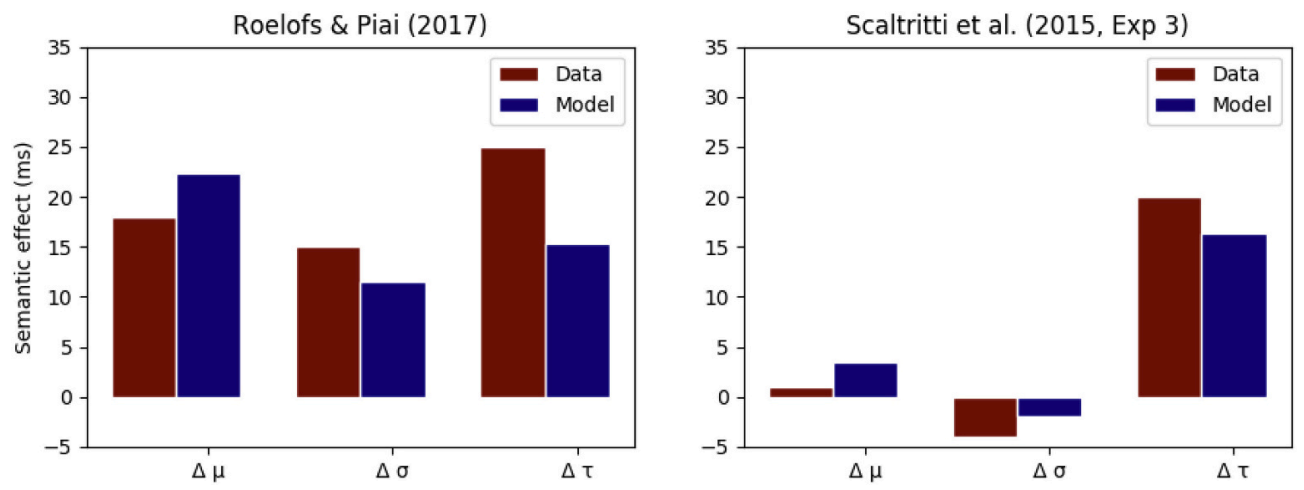

Fig. 2. Delta ex-Gaussian measures of the semantic effect in the model versus empirical data.

It is not implausible that lapses may have occurred more frequently in the study of Roelofs and Piai (2017) than in that of Scaltritti et al. (2015a, 2015b). De Jong, Berendsen, and Cools (1999) provided evidence that a faster pace of stimulus presentation helps participants to remain engaged on the instructed task. A lower lapse rate for Scaltritti et al. than for Roelofs and Piai is in line with this evidence. Roelofs and Piai presented 384 trials to each participant with a trial duration of $4000 \mathrm{~ms}$, whereas there were 480 trials presented by Scaltritti et al. with a mean trial duration of 1711 ms. Thus, on average, the critical trial portion of the task lasted about 1.87 times as long in the experiment conducted by Roelofs and Piai as in the experiment conducted by Scaltritti et al. As a consequence, the participants of Roelofs and Piai were asked to do $80 \%$ as much in about $190 \%$ of the time. To this, we may add that participants in the Scaltritti et al. study enjoyed a self-terminated break after each block of trials, whereas the Roelofs and Piai participants did not have breaks at all. This is likely to have contributed further to differences in engagement, as motivation and energy likely occasionally plummeted in the Roelofs and Piai study, while the Scaltritti et al. participants received periodic opportunities to "reboot" their attentional engagement by disengaging from the task for as long as they considered appropriate. A further reason why participants may have been less engaged in the Roelofs and Piai study is that the number of distinct stimuli in this study was smaller than in Scaltritti et al., and as a consequence, each item was repeated three times. This repetition would have made the task much more tedious and monotonous for the Roelofs and Piai participants, and would have made it harder to sustain their attention for as long as their Scaltritti et al. counterparts. For all of these reasons, it is plausible that attentional lapses were more frequent in the Roelofs and Piai study than in the Scaltritti et al. study.

There are, however, also other ways in which differences between studies may have contributed to the distributional disparities. For example, the stimuli of the two studies are different in terms of the words used, their pictorial representations, and of course, their language. In our simulation work, we observed that lapses magnify inherent conflict. If the stimulus set of Roelofs and Piai happens to elicit a slightly larger semantic interference effect on average, then the difference in lapse rate necessary to explain the disparity between the two studies would be smaller. However, we lack evidence as to whether such a difference in inherent conflict existed and what its magnitude was, so this explanation remains weaker than an explanation in terms of differences in task engagement. Accordingly, while we cannot claim that a difference in lapse rate is the only force driving the observed distributional differences between the two studies, their methodological differences (i.e., number of trials, trial duration, difference in breaks, number of distinct stimuli, number of repetitions) make different lapse rates a plausible explanation. 


\section{Simulation 2: Understanding how lapses affect semantic interference}

\subsection{Aim}

In the second simulation, we sought to gain further insight into the model's behavior so as to obtain an algorithmic understanding of how the duration and frequency of lapses interacts with semantic interference in modulating the shape of the RT distribution. As discussed above, the results of Simulation 1 explain differences in the distributional locus of semantic interference as arising from differences in the three lapse parameter values. While the results suggest that the difference in distributional results was primarily due to the lapse_rate parameter, the overall pattern of differences is somewhat counterintuitive, since not all the differences were in the same direction. In addition, the lack of convergence in the optimization suggested the existence of an irregular parameter space, where several different parameter combinations may be able to yield equivalent results. Thus, it is unclear from these results alone how the three lapse parameters interact with each other, and how these interactions relate to the observed distributional results. The present exploratory simulation sought to shed light on these issues by exploring the model's behavior across a range of parameter configurations. On the one hand, we sought to explore how the individual and joint effects of the three lapse parameters relate to differences in the distributional locus of semantic interference. On the other hand, in order to assess the model's explanatory utility, we sought to determine whether its behavior is qualitatively stable across a range of parameter configurations.

\subsection{Method}

In order to explore the behavior of each lapse parameter and the interactions between them, a grid search was performed on a large region of the parameter space. The grid search included 3 parameter values for the duration_mean and duration_std parameters $(1 \mathrm{~ms}, 100 \mathrm{~ms}$, and $200 \mathrm{~ms}$ ) and 11 values for lapse_rate (0.001, 0.0025, 0.005, 0.0075, $0.010,0.050,0.075,0.10,0.25,0.50$, and 0.75$)$, resulting in a total of 99 parameter configurations. The model was run individually for each parameter combination, following the sampling procedure described in Section 3.

\subsection{Results}

Fig. 3 shows the delta ex-Gaussian estimates of the semantic effect as a function of lapse_rate for each combination of lapse_duration_mean and lapse_duration_stdev. Fig. 4 shows the corresponding decile plots for the same data. Overall, these results show that the total semantic effect $(\Delta \mu$ $+\Delta \tau$, which theoretically corresponds to delta mean RT) increases as lapses become longer and more frequent. Lapse frequency, however, has a stronger impact than lapse length, as evidenced by the fact that the only salient effect of lapse_duration_mean and lapse_duration_stdev in Fig. 3 is the difference between the top left panel and all of the rest. ${ }^{1}$ When it comes to the locus of the semantic effect, however, there are complex interactions between the three lapse parameters. Firstly, in the absence of lapses, the simulation yields WEAVER++'s default semantic interference effect, which is a distributional shift (Roelofs, 2008) that

\footnotetext{
1 Fig. 3 might suggest that the transition between the top left panel and all the others is a step function, and that, consequently, the lapse length parameters play no role in the semantic interference effect once they are set to any value larger than $1 \mathrm{~ms}$. However, we know this not to be the case from our initial exploration of the parameter space. This could have been illustrated in Fig. 3 by increasing the number of intermediate parameter values in the grid search. However, we chose not to do so to avoid an exponential growth in the number of parameter combinations explored.
}

arises from competition at the lemma level. This can be seen in the top left subplots of Figs. 3 and 4, where the mean and standard deviation of lapse duration are both $1 \mathrm{~ms}$ long and thus lapses have almost no effect on semantic interference. The same phenomenon can be observed for the lowest values of lapse_rate throughout the remaining parameter configurations. This is because at extremely low rates, the effect of lapses on semantic interference is also minimal. Thus, in the ideal scenario of perfect or near perfect goal maintenance, the model predicts a baseline semantic interference effect which is not meaningfully influenced by lapses and is completely captured by a distributional shift (i.e., a $\mu$ effect), as in Roelofs (2008). Furthermore, as we discuss later, the results illustrate how lapses have the effect of magnifying this baseline semantic interference effect and modulating its distributional locus. Henceforth, we use the term baseline semantic interference effect to distinguish the pure effect of lexical competition under perfect goal maintenance from the aggregated effect that arises when this baseline effect is magnified by lapses.

Figs. 3 and 4 illustrate how the locus of the semantic effect is most strongly determined by the lapse_rate parameter: At low values of lapse_rate, increasing this parameter causes the bulk of the semantic effect to shift from $\Delta \mu$ to $\Delta \tau$. This is because infrequent lapses have the effect of disproportionately lengthening RT in a small subset of trials, while the vast majority of trials remain unaffected. As a result, the RT distribution becomes highly positively skewed. Since this causes the bulk of the overall interference effect to arise from the contribution of these trials alone, ex-Gaussian analyses correctly attribute a greater proportion of semantic interference to $\tau$, at the expense of $\mu$. At higher values of lapse_rate, however, the pattern is reversed, and further increases in this parameter cause the semantic effect to spill over from $\Delta \tau$ to $\Delta \mu$.

The decile plots in Fig. 4 show how the inversion of this pattern occurs when the slowest decile begins to approach a ceiling level. At even higher rates, the effect of lapses begins to lengthen RT in enough trials so as to influence even the fastest deciles. Therefore, when the frequency of lapses exceeds a certain threshold, the proportion of trials affected by lapses becomes higher than the proportion of unaffected trials, which causes a semantic interference effect that is closer to a distributional shift than to an increase in skewness. Accordingly, this pattern shows up in ex-Gaussian analyses as a larger effect in $\mu$ than in $\tau$. The abovementioned distributional pattern of the lapse_rate parameter holds throughout all configurations of lapse_duration_mean and lapse_duration_stdev, except for the extreme case discussed above where the mean and standard deviation of lapses is only $1 \mathrm{~ms}$ and therefore only the baseline semantic effect can be observed. Thus, in line with the results of Simulation 1, the frequency of lapses seems to be more relevant for the modulation of the semantic effect than their duration. Furthermore, while lapse_duration_mean and lapse_duration_stdev interact with each other, the simulation results show that setting them to the same value does not affect the model's behavior in a qualitatively meaningful way, suggesting that the number of free parameters in the model could be easily reduced by one. We take advantage of this finding in Simulation 3.

\subsection{Discussion}

The simulations showed that the size and distributional locus of the semantic interference effect can be explained by an interaction between all three lapse parameters in the model: lapse_rate, which determines the frequency of lapses, lapse_duration_mean, which determines the mean duration of lapses, and lapse_duration_stdev, which determines the standard deviation of the duration of lapses. According to the model, these lapses modulate the size and distributional locus of the semantic interference effect by magnifying the baseline semantic interference effect, that is, the inherent amount of competition between lexical representations when factoring out the effects of goal maintenance limitations. The model shows a stable pattern of behavior across parameter configurations, which is readily interpretable on a theoretical level. This 




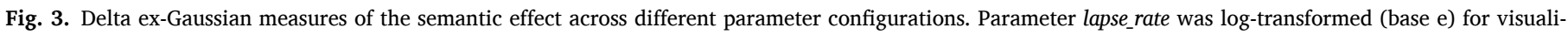

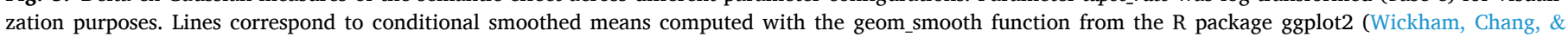
Wickham, 2016). The total effect shown in gray is the sum of the smoothed means of $\Delta \mu$ and $\Delta \tau$.

suggests that the model's ability to replicate the findings in Simulation 1 arises from its architectural properties rather than its flexibility to fit arbitrary functions.

As for the distributional locus of the semantic interference effect, the model predicts a pattern that challenges previous interpretations. Along the lines of the worst performance rule (see Coyle, 2003), Kane and Engle (2003) claimed that "variables thought to affect goal neglect should have a selective effect on skew, and not on the overall distribution shift" (p. 64). While our simulation results formalize and validate their hypothesis that skewing effects can be attributed to lapses of goal maintenance, the results also show how the logical extension of this hypothesis implies that more frequent lapses can also cause distributional shifts by lengthening RT in a larger subset of trials. In fact, the model describes the distributional locus of semantic interference as arising from a continuous tradeoff between $\Delta \mu$ and $\Delta \tau$ which is modulated by the frequency and duration of lapses. While Roelofs and Piai (2017) already argued against the one-to-one mapping between exGaussian parameters and the semantic interference effect, the present simulations go one step further by providing proof-of-principle evidence that the locus of the semantic interference effect can in fact be readily explained as a continuous rather than a discrete phenomenon. Together, the results suggest that Scaltritti et al. (2015a, 2015b) proposal to selectively link ex-Gaussian parameters to cognitive processes (i.e., semantic interference alone) is inherently problematic. In addition, the simulation results suggest that the tradeoff between $\Delta \mu$ and $\Delta \tau$ may be a more informative index of the effects of lapses of goal maintenance on semantic interference than $\Delta \mu$ and $\Delta \tau$ alone. Importantly, in line with
Kane and Engle's (2003) predictions, such a measure should correlate with measures of the cognitive processes that drive lapses. In Simulation 3 , we formalize this hypothesis by predicting a measure of the $\Delta \mu-\Delta \tau$ tradeoff from a single goal_neglect parameter that captures the combined effect of the three lapse parameters.

\section{Simulation 3: Predicting the $\Delta \mu-\Delta \tau$ tradeoff from a single measure of goal maintenance}

\subsection{Aim}

The previous simulation showed the size and locus of the semantic effect to be modulated by the frequency and duration of lapses of goal maintenance. The results suggested that the locus of the semantic interference effect moves along a $\Delta \mu-\Delta \tau$ tradeoff continuum as a function of attentional performance. Nevertheless, the model used in Simulations 1 and 2 is limited in its ability to make straightforward predictions regarding goal maintenance, since the effect of lapses is driven by three separate parameters. The aim of the third simulation was to formalize the aforementioned hypothesis by predicting a measure of the $\Delta \mu-\Delta \tau$ tradeoff from a single unifying measure of goal maintenance. To this end, a goalneglect parameter was introduced which determines the behavior of all three lapse parameters. In line with the results of Simulation 2, we predicted that the correlation between the goalneglect parameter and the $\Delta \mu-\Delta \tau$ tradeoff should be U-shaped. 


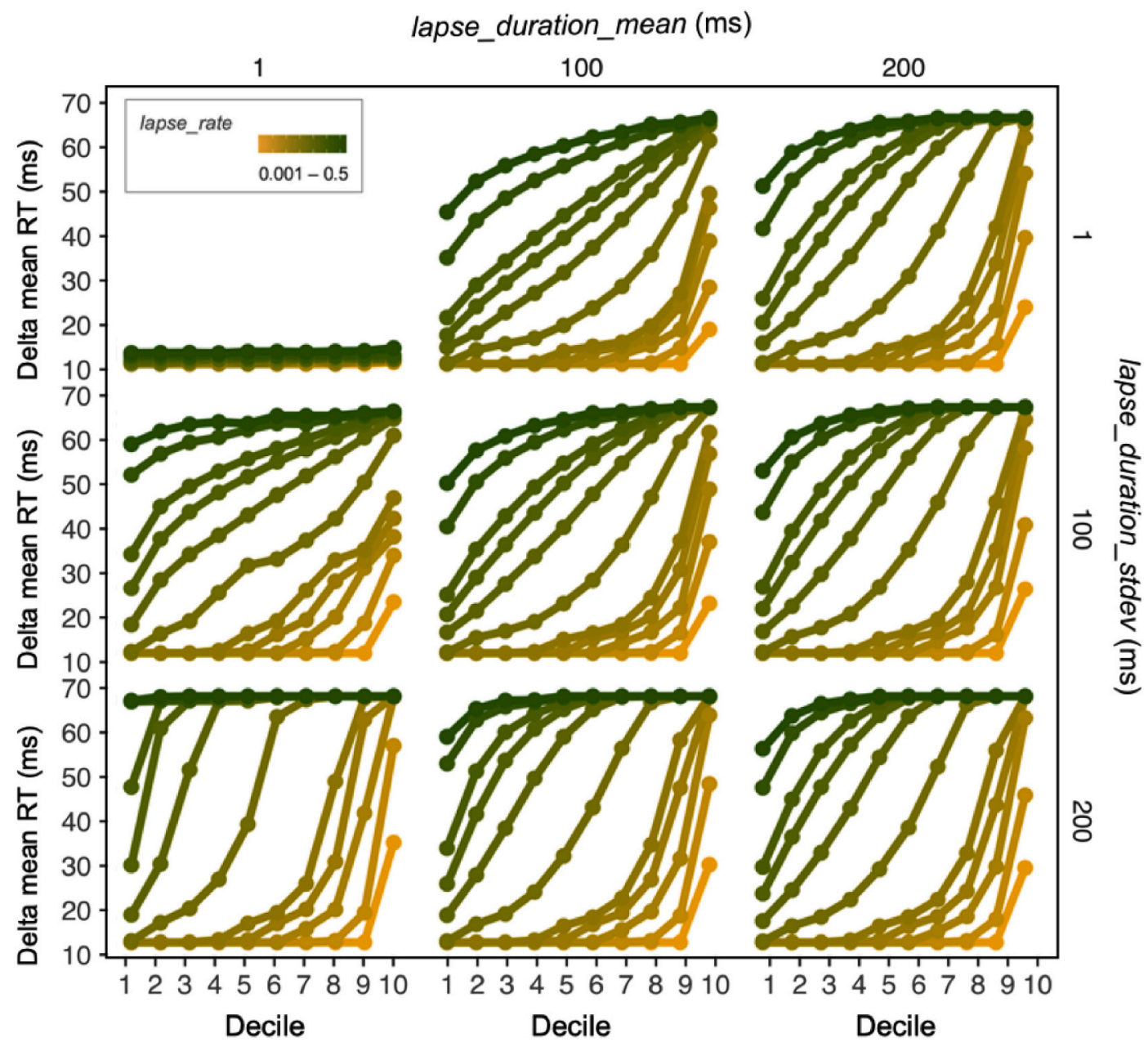

Fig. 4. Delta decile plots of the semantic effect across different parameter configurations.

\subsection{Method}

The $\Delta \mu-\Delta \tau$ tradeoff was operationalized by subtracting $\Delta \tau$ from $\Delta \mu$. The new goal_neglect parameter was introduced in the model to represent limitations of goal maintenance as a single construct. The value of each lapse parameter was made to be a function of the goal_neglect parameter (e.g., lapse_rate' $=$ goal_neglect * lapse_rate). Thus, this parameter has the function of scaling the three lapse parameters and making them covary. As a result, the goal_neglect parameter can be interpreted as a single measure of the effect of lapses of goal maintenance, and can therefore be used to make more straightforward and readily interpretable predictions than the three separate lapse parameters. For the present simulation, a one-dimensional grid search was performed on the goal_neglect parameter alone. Based on the results of Simulation 2 that setting lapse_duration_mean and lapse_duration_stdev to the same value does not affect the model's qualitative behavior, they were fixed to have same value across configurations of the goalneglect parameter. As a result, the addition of the goal_neglect parameter does not increase the degrees of freedom relative to Simulations 1 and 2. In the present simulation, a grid search was carried out exploring 18 values of the goal_neglect parameter, ranging from 0.01 to 1 . The values of lapse_duration_mean and lapse_duration_stdev were set to $1000 \mathrm{~ms}$, and lapse_rate was set to 0.1 . Since the goal of this simulation is to summarize the findings of Simulations 1 and 2 with a single measure of goal neglect, we selected parameter values which, once scaled by the goal_neglect parameter, yielded configurations of the lapse parameters comparable to those reported in Simulations 1 and 2 . These values were then multiplied by each of the 18 values of the goalneglect parameter. As a result of this scaling, the lowest value of goal_neglect yielded a lapse_rate of 0.001 and a lapse_duration_mean and lapse_duration_stdev of $100 \mathrm{~ms}$, while the highest value of goal_neglect explored yielded a lapse_rate of 0.1 and a lapse_duration_mean and lapse_duration_stdev of $1000 \mathrm{~ms}$.

\subsection{Results and discussion}

The results are shown in Fig. 5, where the $\Delta \mu-\Delta \tau$ tradeoff is plotted as a function of goalneglect. The figure illustrates how the pattern of the goalneglect parameter mimics the behavior of the lapse_rate parameter in showing a gradual reversal of the relative size of $\Delta \mu$ with respect to $\Delta \tau$. This is in line with the results from Simulations 1 and 2 that the distributional locus of the semantic interference effect is more strongly determined by the frequency of lapses than by their duration. More importantly, as predicted, the model showed this relationship to be Ushaped. That is, in line with the results of Simulation 2, the model predicts that low goal neglect (i.e., high goal maintenance) should cause the semantic effect to be more present in $\Delta \mu$, whereas increasingly higher goal neglect will cause the effect to spill over from $\Delta \mu$ to $\Delta \tau$, and even higher degrees of goal neglect should cause it to spill over from $\Delta \tau$ back to $\Delta \mu$. By representing the effect of lapses as a single goal neglect variable, the model now makes a clear prediction about the correlational patterns that should be found between ex-Gaussian parameters and participants' attentional performance.

Importantly, the model's prediction is consistent not only with the PWI findings we have discussed so far, but also with findings from regular picture naming studies. For example, Shao, Roelofs, and Meyer (2012) found a correlation between working memory capacity 


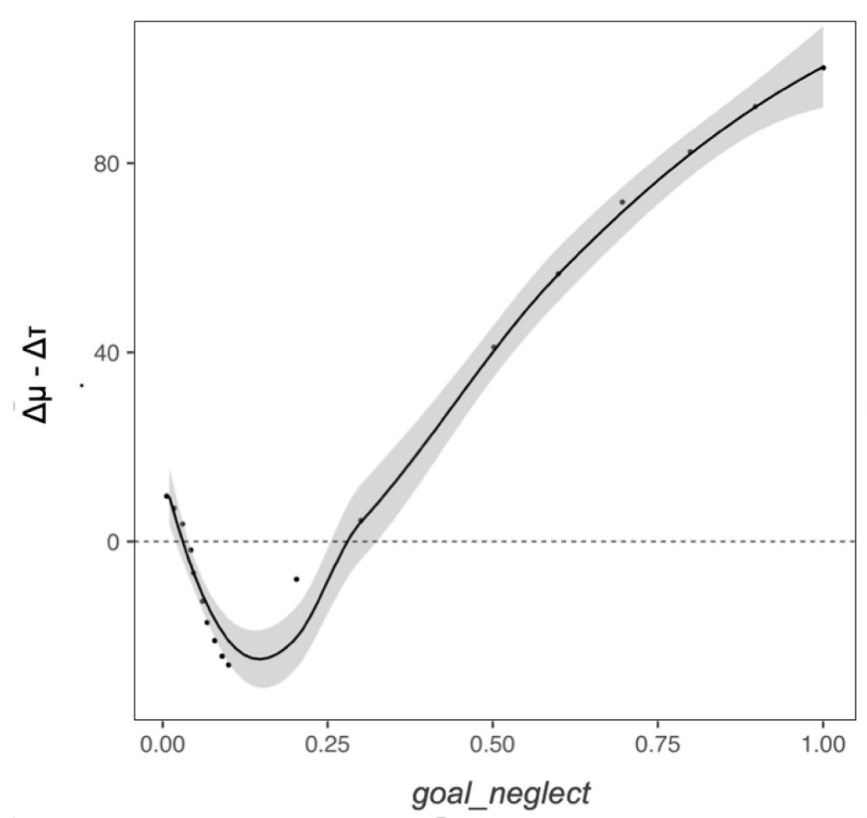

Fig. 5. $\Delta \mu-\Delta \tau$ tradeoff as a function of goal_neglect. The line corresponds to the smoothed mean of the $\Delta \mu-\Delta \tau$ tradeoff computed with the geom_smooth function from the R package ggplot2 (Wickham et al., 2016).

(operationalized as Operation Span) and the $\tau$ parameter of RT in a regular picture naming task. The $\mu$ parameter, on the other hand, did not correlate with Ospan in either condition. Importantly, while Ospan correlated with the $\tau$ parameter in both action and object naming, its correlation with mean RT was restricted to action naming. This pattern is readily accounted for in the model as arising from a spill-over effect from $\tau$ to $\mu$, as a consequence of the interaction between lapses of goal maintenance and the amount of competition between lexical representations (i.e., the baseline semantic interference effect). Assuming that action naming causes more lexical competition than object naming (Shao et al., 2012; Shao, Roelofs, Acheson, \& Meyer, 2014), the baseline semantic effect in action naming would have been magnified by lapses to a greater extent than the semantic effect in object naming, just like our simulations showed lapses to magnify the interference effect of related distractors to a higher degree than the interference effect of unrelated distractors. Thus, the findings of Shao et al. (2012) highlight the explanatory utility of our model, as they can be readily explained as the effect of a continuous spill-over from $\tau$ to $\mu$.

Additional evidence for a $\mu-\tau$ trade-off in picture naming comes from a study by Jongman et al. (2015, Experiment 2), which explored the role of sustained attention in speech production. In this experiment, participants performed object naming in a single task versus a dual task setting. It was found that sustained attention (as measured by a visual digit discrimination task) correlated with the $\tau$ parameter of object naming RT in both conditions. On the other hand, $\mu$ did not significantly correlate with object naming in the single task condition, but approached significance in the dual task condition. This was interpreted as dual tasking taking a greater toll on sustained attention than single tasking. Importantly, these findings are also in line with our model's prediction that increasing attentional demands should cause semantic competition effects to spill over from $\tau$ to $\mu$, as they cause longer and more frequent lapses. In sum, our simulation results predict a U-shaped relationship between attentional engagement and a $\Delta \mu-\Delta \tau$ tradeoff, and this pattern is compatible with experimental findings involving different picture naming variants and different attentional measures that can be related to task goal maintenance.

\section{Behavioral experiment}

In our simulations, we showed that our model can explain differences in the size and distributional locus of the semantic interference effect as arising from differences in attentional engagement alone. This insight, however, can only be considered a proof-of-principle demonstration, given the post-hoc nature of our simulations and the existence of several differences between the studies we have considered. We thus set out to test this hypothesis empirically with a new behavioral experiment where attentional engagement is manipulated within-subjects. In particular, we used monetary reward as a proxy for motivation (see Woodworth, 1938, and Woodworth \& Schlosberg, 1954, for discussion of an early study on how incentives influence RT distributions).

Participants completed a picture-word interference task. A prestimulus cue informed the participants about whether the upcoming trial would carry low $(€ 0.01)$ or high (€0.10) reward. If the participant's response was correct and fell within a pre-specified RT range, the trial was scored as correct, and its associated reward was added to the participant's final payment. In contrast, if the response was incorrect (e.g., wrong word) or fell outside the permitted RT range, the trial was penalized, and its associated reward was subtracted from the final payment rather than added to it. We predicted that participants would be more engaged in the high-reward condition and, as a result, would be less affected by attentional fluctuations (i.e., the lapses in our model). As a result, higher reward should decrease the size of the semantic interference effect and affect its locus in the distribution. As discussed in Section 3 on Simulation 1, the model cannot a priori predict which exact distributional patterns we are likely to observe. That is, the model cannot reliably predict, for example, whether one of our conditions should yield an effect in $\tau$ alone (as in Scaltritti et al., 2015a, 2015b) or both in $\mu$ and $\tau$ (as in Roelofs \& Piai, 2017). Furthermore, as shown in Simulations 2 and 3, our simulations warn against formulating hypotheses in terms of absolute mappings between the semantic effect and individual ex-Gaussian parameters. As our simulations illustrate, this approach would allow for genuine differences to go undetected, and thus severely compromise the statistical power of our study. Consequently, we limited ourselves to predicting that the reward manipulation should yield a significant decrease in the magnitude of the semantic interference effect in at least one of the two ex-Gaussian parameters of interest, either $\mu$ or $\tau$. If reward significantly modulates the effect of semantic interference in both $\mu$ and $\tau$, then the effect of reward on $\mu$ and $\tau$ should be in the opposite direction (i.e., higher interference in $\tau$ and lower interference in $\mu$ or vice versa), in line with the U-shaped $\Delta \mu-\Delta \tau$ tradeoff shown in Simulation 3. Finally, we predict that our reward manipulation should decrease RT across distractor conditions, reflecting the fact that attentional lapses should slow down responses even in low conflict conditions (i.e., unrelated distractor).

\subsection{Method}

\subsubsection{Participants}

The experiment was carried out with 41 native speakers of Dutch recruited through the Max Planck Institute's database. The data from one participant was excluded for not complying with the instructions. Participants were paid on the basis of their performance, whereby the reward of each correct trial was added to the final payment and the reward of each penalized trial was subtracted from it. The payment ranged from a minimum of $€ 10$ to a maximum of $€ 20$.

\subsubsection{Design and materials}

The variables reward (low, high) and distractor word (semantically related, unrelated) were fully crossed in a $2 \times 2$ within-subjects design. The stimuli used as low- and high-reward cues were color pictures of a one-cent and a ten-cent Euro coin, respectively. The picture-word materials were taken from the study of Roelofs and Piai (2017). These consist of 32 line-drawings of common objects, each paired with a 
semantically related or unrelated Dutch word, yielding 64 different pairs. As in Roelofs and Piai, each picture-word pair was repeated three times per condition, yielding a total of 384 trials. All the visual stimuli, including the reward cues, were adjusted to fit in a $10 \times 10 \mathrm{~cm}$ white frame. The stimuli were presented in semi-random order, with the constraint that pictures and words were not repeated on successive trials.

\subsubsection{Procedure}

Participants sat individually in front of a computer screen and PC equipped with a voice key. The experiment began with a familiarization phase where participants saw each drawing together with the name of the depicted object that they would be asked to provide during the experiment. Participants then received written instructions and completed a practice phase which was identical to the main procedure, except that it consisted of 20 filler trials. During the practice phase, the experimenter corrected any errors in performance and adjusted the voice key if necessary. Participants were then encouraged to ask questions about the procedure. Next, the actual experiment began, which consisted of 384 trials divided into 6 blocks. Participants were instructed to name the picture and ignore the distractor. They were told that both being slow and inaccurate (saying the wrong word) would incur a penalty, and thus they should put equal emphasis on speed and accuracy.

The trial structure is illustrated in Fig. 6. Each trial began with a fixation cross, which remained on the screen for $200 \mathrm{~ms}$. This was followed by a blank screen $(200 \mathrm{~ms})$ and the reward cue, which remained on the screen for $1400 \mathrm{~ms}$. This was followed by a blank screen $(200 \mathrm{~ms})$, a fixation point $(200 \mathrm{~ms})$, and an additional blank screen $(200 \mathrm{~ms})$. The picture was then presented for $1500 \mathrm{~ms}$ or until a response was recorded by the voice key. Therefore, there was a period of $2 \mathrm{~s}$ from the onset of the reward cue to the onset of the picture-word stimulus, during which participants were informed about the reward of the upcoming trial and could prepare accordingly. The inter-trial interval was $2 \mathrm{~s}$.

After each block, participants received feedback on their performance: They received information about the amount of money they had earned during the block (the sum of the reward from all correct trials) and the amount of money they had lost (the sum of all penalized trials). Money earned was shown in green, preceded by a plus sign. Money lost was shown in red, preceded by a minus sign. These quantities were presented simultaneously in the middle of the screen, with earned reward on the top and lost reward on the bottom. This feedback was calculated as follows: Speed penalties were computed automatically from voice-key responses, whereby responses outside a given range were penalized. The lower bound was $200 \mathrm{~ms}$, so that false starts or the accidental triggering of the voice key would be penalized. The upper bound was calculated by rounding up the 75th percentile from Roelofs and Piai (2017), so as to make sure that roughly $75 \%$ of the trials were unlikely to be penalized based on speed. Our aim was to have a penalty bound that was realistically strict, yet not tough enough to demotivate participants. Errors were coded online by the experimenter and inputted manually. The feedback from each block remained on the screen for $30 \mathrm{~s}$. There were no breaks between blocks. The session lasted for approximately an hour.

\subsubsection{Analysis}

RTs were computed graphically using the Praat software. Errors were annotated offline. Trials in which the participant produced the wrong word, self-corrected, or triggered the voice key by accident were considered errors and excluded from the analysis.

Raw RTs were submitted to ex-Gaussian analyses using the mexgauss function from the retimes $\mathrm{R}$ package (Massidda, 2013). These analyses were performed individually for each participant and condition. The resulting ex-Gaussian estimates were submitted to three separate linear regressions (one per ex-Gaussian parameter). Each regression model included as fixed effects the sum-coded predictors reward (low $=-1$, high $=1$ ) and distractor (unrelated $=-1$, related $=1$ ), plus the interaction between the two. The resulting R syntax was as follows: Parameter $\sim$ Distractor * Reward.

Previous studies such as Roelofs and Piai (2017) and Scaltritti et al. (2015a, 2015b) complemented their statistical analyses of ex-Gaussian estimates with an analogous analysis of mean RT estimates. While this makes the analyses more comparable, aggregating over trials inevitably leads to a loss of information. Since the distractor effect is typically quite small and we were looking to detect its interaction with a presumably much smaller effect (as per our model's predictions), we sought to maximize our statistical power by exploiting every single trial in the data set. We therefore submitted RTs to mixed-effects linear regression. For this analyses, raw RTs were log-transformed so as to reduce skew (Baayen, Davidson, \& Bates, 2008). The fixed terms in the model were the same sum-coded variables as before. The inclusion of random terms was informed by a model comparison based on the Akaike Information Criterion (Akaike, 1973). The winner was a model that included only random intercepts for items. However, since our study examined attention, and its relevance for individual differences is well documented in the literature, we opted for the second-best model, which



Fig. 6. Trial structure in our experiment. 
included intercepts for participants as well. The resulting $\mathrm{R}$ syntax was the following: Log_RT Distractor * Reward + (1 | Participant $)+(1 \mid$ Picture).

\subsection{Results}

Table 2 shows mean RT, mean error, and ex-Gaussian parameter estimates per condition. Fig. 7 shows mean RT by decile for each reward condition. These plots were computed by rank-ordering RTs into 10 bins and computing the mean of each bin individually for each condition and participant. Deciles were then averaged across participants for each condition. As Fig. 7 shows, high reward yielded slightly faster responses than low reward, but this difference between conditions was restricted to the slowest trials, i.e., the tail of the distribution. Fig. 8 shows mean RT by decile per distractor condition. As we can see, related distractors yielded slower responses than unrelated distractors. While this difference can be observed throughout the distribution, it became more salient for the slowest deciles. Fig. 9 gives the distractor effect (semantically related - unrelated) by reward condition. This was calculated separately for each reward condition by subtracting the deciles in the unrelated condition from the deciles in the related condition. The resulting delta deciles were then averaged across participants. The plot shows how the distractor effect grew as a function of RT in both conditions, but this growth was smaller and slower in the high reward condition. As a result, the difference in the distractor effect between the high and low reward conditions is also largest in the tail of the distribution. Finally, errors were not submitted to inferential analyses due to their low frequency in the dataset. However, as shown in Table 2, the observed pattern appears to be consistent with the mean RT results, with

Table 2

Mean RT, mean error rate, and ex-Gaussian estimates per condition. The standard error is shown in brackets.

\begin{tabular}{|c|c|c|c|c|c|c|}
\hline Distractor & Reward & $\begin{array}{l}\text { Mean } \\
\text { RT }\end{array}$ & $\tau$ & $\mu$ & $\sigma$ & $\begin{array}{l}\text { Mean } \\
\text { error }\end{array}$ \\
\hline Related & High & $\begin{array}{l}652 \\
(47)\end{array}$ & $\begin{array}{l}105.15 \\
(25.25)\end{array}$ & $\begin{array}{l}546.36 \\
(39.52)\end{array}$ & $\begin{array}{l}51.4 \\
(20.43)\end{array}$ & $\begin{array}{l}6.1 \\
(4.4)\end{array}$ \\
\hline Unrelated & High & $\begin{array}{l}635 \\
(42)\end{array}$ & $\begin{array}{l}92.55 \\
(27.71)\end{array}$ & $\begin{array}{l}540.2 \\
(36.8)\end{array}$ & $\begin{array}{l}44.08 \\
(19.06)\end{array}$ & $\begin{array}{l}3.7 \\
(3.1)\end{array}$ \\
\hline Related & Low & $\begin{array}{l}667 \\
(55)\end{array}$ & $\begin{array}{l}121.27 \\
(22.63)\end{array}$ & $\begin{array}{l}544.09 \\
(52.33)\end{array}$ & $\begin{array}{l}52.54 \\
(29.45)\end{array}$ & $\begin{array}{l}6.3 \\
(4.9)\end{array}$ \\
\hline Unrelated & Low & $\begin{array}{l}633 \\
(46)\end{array}$ & $\begin{array}{l}90.04 \\
(27.95)\end{array}$ & $\begin{array}{l}540.54 \\
(44.54)\end{array}$ & $\begin{array}{l}47.44 \\
(20.42)\end{array}$ & $\begin{array}{l}4.2 \\
(3.4)\end{array}$ \\
\hline
\end{tabular}

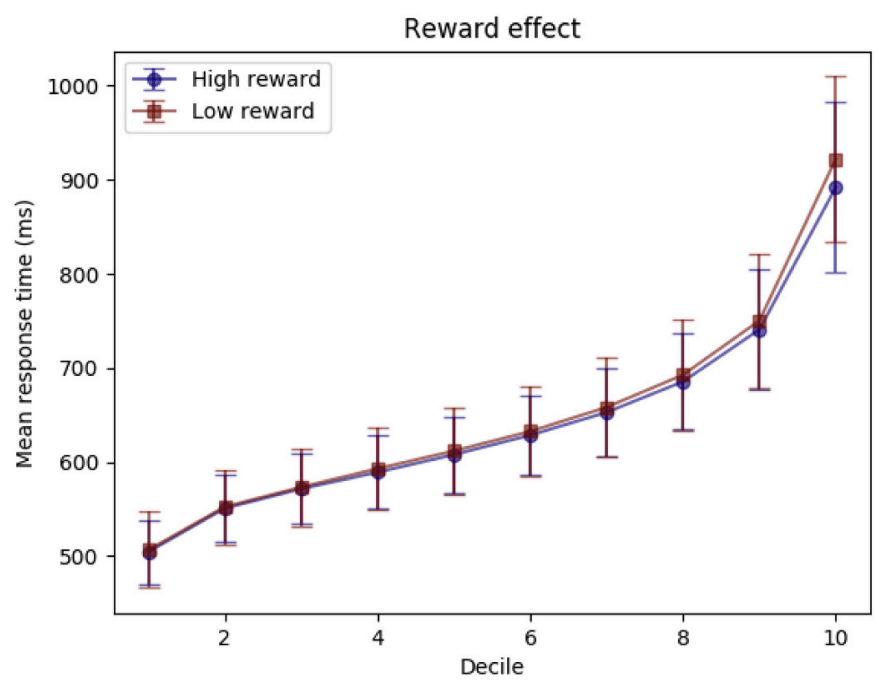

Fig. 7. Decile plot for the high and low reward conditions. The error bars indicate standard error.

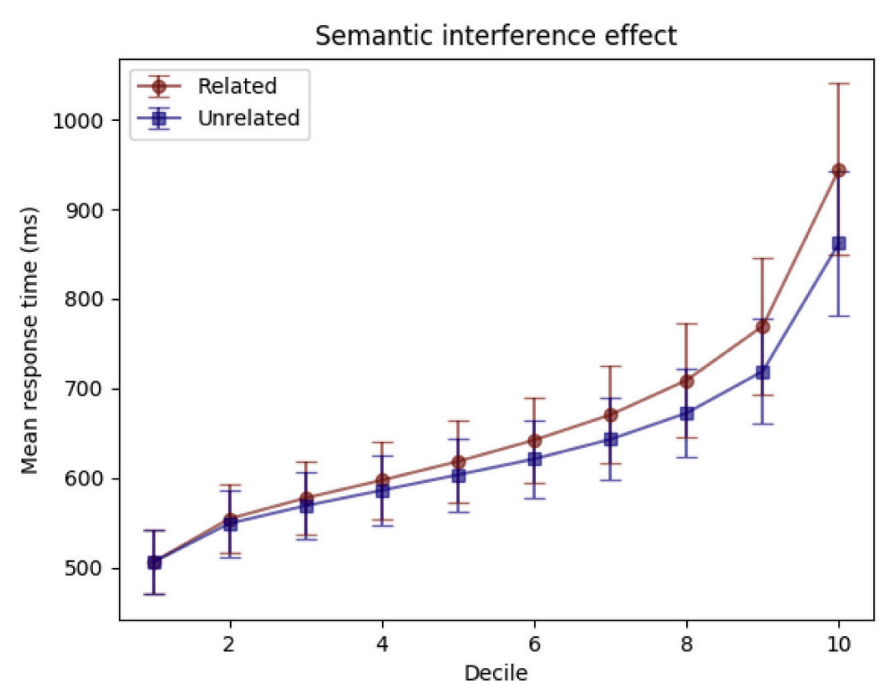

Fig. 8. Decile plot for the related and unrelated distractor conditions. The error bars indicate standard error.

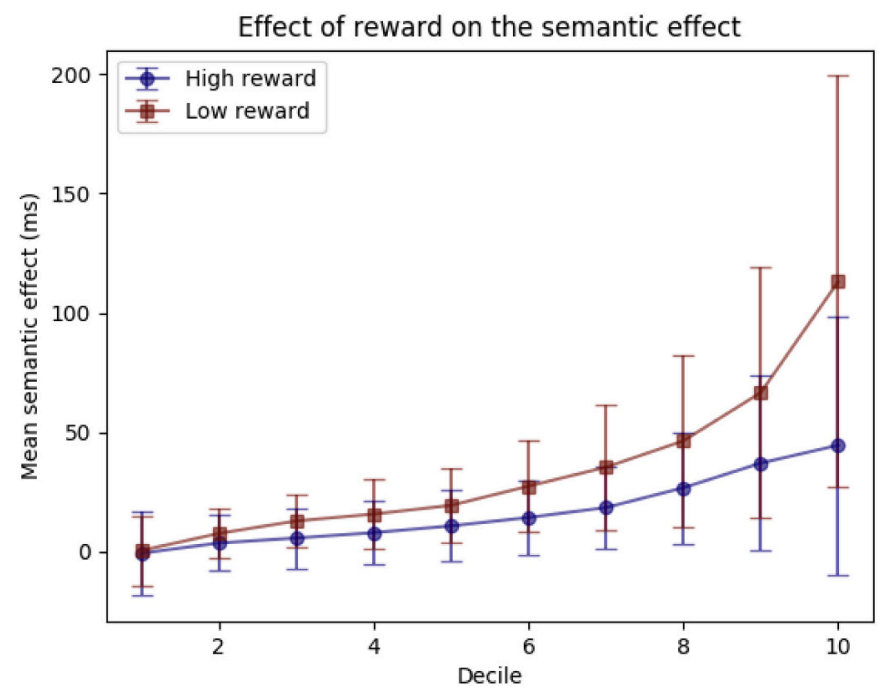

Fig. 9. Semantic effect (related - unrelated deciles) for the high and low reward conditions. The error bars indicate standard error.

error rate being increased by semantic interference and reduced by reward.

\subsubsection{Distributional analyses}

The regression coefficients from the distributional analyses are presented in Tables 3 (for $\mu$ ), 4 (for $\tau$ ), and 5 (for $\sigma$ ). These analyses revealed no significant effect of reward for any of the three ex-Gaussian parameters. The distractor effect was highly significant for $\tau$ and approached significance for $\sigma$, but not for $\mu$. Finally, the interaction between reward and distractor was significant for $\tau$, but not for $\mu$ or $\sigma$.

Table 3

Regression coefficients for the $\mu$ analysis.

\begin{tabular}{lllll}
\hline & $\beta$ & SE & $t$-value & $p(>|\mathrm{t}|)$ \\
\hline Intercept & 542.80 & 3.50 & 155.15 & $<0.001 * * *$ \\
Distractor & 2.43 & 3.50 & 0.70 & 0.49 \\
Reward & 0.49 & 3.50 & 0.14 & 0.89 \\
Distractor $\times$ Reward & 0.65 & 3.50 & 0.19 & 0.85 \\
\hline
\end{tabular}


Table 4

Regression coefficients for the $\tau$ analysis.

\begin{tabular}{lllll}
\hline & $\beta$ & SE & $t$-value & $p(>|\mathrm{t}|)$ \\
\hline Intercept & 102.25 & 2.08 & 49.18 & $<0.001 * * *$ \\
Distractor & 10.96 & 2.08 & 5.27 & $<0.001 * * *$ \\
Reward & -3.40 & 2.08 & -1.64 & 0.10 \\
Distractor $\times$ Reward & -4.66 & 2.08 & -2.24 & $0.03 *$ \\
\hline
\end{tabular}

Table 5

Regression coefficients for the $\sigma$ analysis.

\begin{tabular}{lllll}
\hline & $\beta$ & $\mathrm{SE}$ & $t$-value & $p(>|\mathrm{t}|)$ \\
\hline Intercept & 48.86 & 1.82 & 26.87 & $<0.001 * * *$ \\
Distractor & 3.11 & 1.82 & 1.71 & 0.09 \\
Reward & -1.12 & 1.82 & -0.62 & 0.54 \\
Distractor $\times$ Reward & 0.56 & 1.82 & 0.31 & 0.76 \\
\hline
\end{tabular}

Table 6

Regression coefficients for the log RT analysis.

\begin{tabular}{lllll}
\hline & $\beta$ & SE & $t$-value & $p(>|\mathrm{t}|)$ \\
\hline Intercept & 6.455 & 0.016 & 409.768 & $<0.001^{* * *}$ \\
Distractor & 0.019 & 0.001 & 14.932 & $<0.001^{* * *}$ \\
Reward & -0.004 & 0.001 & -2.778 & $0.006^{* * *}$ \\
Distractor $\times$ Reward & -0.005 & 0.001 & -3.930 & $<0.001^{* * *}$ \\
\hline
\end{tabular}

\subsection{2. $\log R T$ analysis}

The model coefficients for the analysis on log RTs are shown in Table 6. This analysis revealed a significant effect of reward and a highly significant effect of distractor. Finally, the interaction between reward and distractor was highly significant.

\subsection{Discussion}

We carried out a PWI experiment to test the hypothesis that the attentional engagement of participants can modulate the size and distributional properties of the semantic interference effect. Scaltritti et al. (2015a, 2015b) observed a semantic effect only in the tail of the RT distribution ( $\tau$ parameter), whereas Roelofs and Piai (2017) found a larger semantic effect which showed up in both the tail of the distribution $(\tau)$ and its leading edge $(\mu)$. Our simulation work offered proofof-principle evidence that these differences between studies could be explained as arising from a difference in attentional engagement. In the present study, we manipulated attentional engagement by offering participants either high $(€ 0.10)$ or low $(€ 0.01)$ monetary reward upon sufficient performance on each corresponding trial. According to the model, the baseline semantic interference effect, however small, should be present across the entire distribution ( $\mu$ effect). In addition, lapses of attentional engagement should inflate the semantic effect and modulate its distributional dynamics, so that relatively infrequent lapses would inflate the tail of the distribution, but relatively frequent lapses would begin to inflate $\mu$, as more and more trials become affected. Therefore, our reward manipulation should have an asymmetric effect on $\mu$ and $\tau$. That is, the reward effect should either show up in one parameter alone or exhibit the opposite trend in each of them (e.g., decrease $\tau$ but increase $\mu$ ). Our results are consistent with these predictions: In our experiment, high reward yielded a significantly smaller semantic interference effect than did low reward. And this difference was restricted to the $\tau$ parameter.

Compared to the Roelofs and Piai (2017) experiment, which used the same stimuli, our participants were overall faster, and the semantic interference effect was overall smaller. In addition, Roelofs and Piai found the semantic effect in both $\mu$ and $\tau$, whereas we found it only in the $\tau$ parameter. All of these differences between the two studies can be accounted for by our model by assuming that the participants of the present experiment had higher attentional engagement. This is in fact extremely likely, given that our study involved some degree of reward on all trials, and therefore participants' motivation to remain attentive should be higher than in the experiment of Roelofs and Piai across the board. Additionally, we penalized RTs above the 75th percentile of Roelofs and Piai's data, which is likely to have led participants to aim for faster performance overall.

In sum, our results confirmed the model predictions that boosting attentional engagement not only decreases RT across the board, but also decreases the semantic interference effect and alters its distributional properties in a way that is consistent with our $\Delta \mu-\Delta \tau$ trade-off hypothesis. Furthermore, the differences between our study and Roelofs and Piai (2017) are all consistent with the model predictions, which provides additional support for the hypothesis discussed in Simulation 1 that differences between previous studies may have also partly been caused by differences in attentional engagement.

\section{Summary and conclusions}

Previous evidence from distributional analyses of RTs in regular picture naming and picture-word interference showed that attention plays an important role in spoken word production. However, there was still no computational account of how properties of RT distributions reflect the effect of attentional processes and how these may modulate the amount of conflict between lexical representations. To fill this gap, we presented an extension of the WEAVER ++ model in which lapses of attention allow for lexical conflict to build up unsupervised on a subset of trials, thus modulating the shape of the resulting RT distribution. Computer simulations revealed that our model resolves discrepancies between outcomes of studies on semantic interference in the literature. Moreover, the model's predictions were confirmed in a new experiment where the motivation of participants to remain attentive determined the size and distributional locus of semantic interference in picture naming. Our model therefore provides a useful framework for interpreting distributional analyses of RT data in picture naming tasks. We conclude that the process modeling of RT distributions provides valuable insight into the interplay between attention and conflict in spoken word production.

\section{Declaration of Competing Interest}

We have no conflicts of interest to disclose.

\section{Appendix A}

\section{Simulation 1 methods}

The model parameters were optimized using Simulated Annealing (Van Laarhoven \& Aarts, 1987). The objective function to be minimized was the root mean squared error (RMSE) between the model's output and the empirical data. This measure was calculated on the delta deciles of the semantic interference effect (see Section 2.4). The optimization process was carried out in two steps. In the first step, the objective function was calculated on the mean of the two empirical data sets. For this optimization problem, all the model parameters were allowed to vary, so as to find the parameter 
configuration that is most likely to explain the experimental findings of both experiments. The resulting parameter configuration (presented as Appendix B) was used as the starting point for the second optimization step. In this second step, two separate models were fitted, one for each of the two data sets. For these simulations, only the three lapse parameters were allowed to vary. As a result, any difference in the distributional behavior of the two models can be attributed solely to the behavior of the lapse parameters. For each of the abovementioned optimization problems, the algorithm was run ten times. The results were then collapsed across runs and the best performing parameter configuration was selected on the basis of RMSE.

\section{Appendix B}

Common parameters used in Simulation 1

\begin{tabular}{ll}
\hline parameter & value \\
\hline distractor_duration $(\mathrm{ms})$ & 106 \\
sem_rate & 0.0071596 \\
lem_rate & 0.0598442 \\
decay_rate & 0.0342529 \\
extin & 0.2480972 \\
crit_diff & 19.183263 \\
\hline
\end{tabular}

\section{References}

Akaike, H. (1973). Information theory and an extension of the maximum likelihood principle. In B. N. Petrov, \& F. Csaki (Eds.), Proceedings of the second international symposium on information theory (pp. 267-281). Budapest: Akademiai Kiado.

Baayen, R. H., Davidson, D. J., \& Bates, D. M. (2008). Mixed-effects modeling with crossed random effects for subjects and items. Journal of Memory and Language, 59 (4), 390-412.

Balota, D. A., Yap, M. J., Cortese, M. J., \& Watson, J. M. (2008). Beyond mean response latency: Response time distributional analyses of semantic priming. Journal of Memory and Language, 59(4), 495-523.

Coyle, T. R. (2003). A review of the worst performance rule: Evidence, theory, and alternative hypotheses. Intelligence, 31(6), 567-587.

Damian, M. F., \& Martin, R. C. (1999). Semantic and phonological codes interact in single word production. Journal of Experimental Psychology: Learning, Memory, and Cognition, 25(2), 345-361.

De Jong, R., Berendsen, E., \& Cools, R. (1999). Goal neglect and inhibitory limitations: Dissociable causes of interference effects in conflict situations. Acta Psychologica, 101 (2-3), 379-394.

James, W. (1890). The principles of psychology. New York: Holt.

Jongman, S. R., Roelofs, A., \& Lewis, A. G. (2020). Attention for speaking: Prestimulus motor-cortical alpha power predicts picture naming latencies. Journal of Cognitive Neuroscience, 32(5), 747-761.

Jongman, S. R., Roelofs, A., \& Meyer, A. S. (2015). Sustained attention in language production: An individual differences investigation. Quarterly Journal of Experimental Psychology, 68(4), 710-730.

Kane, M. J., \& Engle, R. W. (2003). Working-memory capacity and the control of attention: The contributions of goal neglect, response competition, and task set to Stroop interference. Journal of Experimental Psychology: General, 132, 47-70.

Kleinman, D. (2013). Resolving semantic interference during word production requires central attention. Journal of Experimental Psychology. Learning, Memory, and Cognition, 39(6), 1860-1877.

Levelt, W. J., Roelofs, A., \& Meyer, A. S. (1999). A theory of lexical access in speech production. Behavioral and Brain Sciences, 22(1), 1-38.

Luce, R. D. (1986). Response times: Their role in inferring elementary mental organization. New York: Oxford University Press.

MacLeod, C. M. (1991). Half a century of research on the Stroop effect: An integrative review. Psychological Bulletin, 109(2), 163-203.

Massidda, D. (2013). Retimes: Reaction time analysis. R package version 0.1.2. https://CRAN.R-project.org/package=retimes.

Piai, V., Roelofs, A., \& Schriefers, H. (2014). Locus of semantic interference in picture naming: Evidence from dual-task performance. Journal of Experimental Psychology: Learning, Memory, and Cognition, 40(1), 147-165.

Roelofs, A. (1992). A spreading-activation theory of lemma retrieval in speaking. Cognition, 42(1-3), 107-142.
Roelofs, A. (2003). Goal-referenced selection of verbal action: Modeling attentional control in the Stroop task. Psychological Review, 110, 88-125.

Roelofs, A. (2008). Dynamics of the attentional control of word retrieval: Analyses of response time distributions. Journal of Experimental Psychology: General, 137(2), $303-323$.

Roelofs, A. (2018). A unified computational account of cumulative semantic, semantic blocking, and semantic distractor effects in picture naming. Cognition, 172, 59-72.

Roelofs, A., \& Hagoort, P. (2002). Control of language use: Cognitive modeling of the hemodynamics of Stroop task performance. Cognitive Brain Research, 15, 85-97.

Roelofs, A., \& Piai, V. (2017). Distributional analysis of semantic interference in picture naming. Quarterly Journal of Experimental Psychology, 70(4), 782-792.

Scaltritti, M., Navarrete, E., \& Peressotti, F. (2015a). Distributional analyses in the picture-word interference paradigm: Exploring the semantic interference and the distractor frequency effects. Quarterly Journal of Experimental Psychology, 68(7), 1348-1369.

Scaltritti, M., Navarrete, E., \& Peressotti, F. (2015b). Distributional analyses in the picture-word interference paradigm: Exploring the semantic interference and the distractor frequency effects. Unpublished raw data [dataset].

Schnur, T. T., \& Martin, R. (2012). Semantic picture-word interference is a postperceptual effect. Psychonomic Bulletin \& Review, 19(2), 301-308.

Schriefers, H., Meyer, A. S., \& Levelt, W. J. (1990). Exploring the time course of lexical access in language production: Picture-word interference studies. Journal of Memory and Language, 29(1), 86-102.

Shao, Z., Roelofs, A., Acheson, D. J., \& Meyer, A. S. (2014). Electrophysiological evidence that inhibition supports lexical selection in picture naming. Brain Research, 1586, $130-142$.

Shao, Z., Roelofs, A., \& Meyer, A. S. (2012). Sources of individual differences in the speed of naming objects and actions: The contribution of executive control. Quarterly Journal of Experimental Psychology, 65(10), 1927-1944.

Van Laarhoven, P. J., \& Aarts, E. H. (1987). Simulated annealing. In P. J. van Laarhoven, \& E. H. Aarts (Eds.), Simulated annealing: Theory and applications (pp. 7-15). Dordrecht: Springer.

Wickham, H., Chang, W., \& Wickham, M. H. (2016). Package "ggplot2". Create Elegant Data Visualisations Using the Grammar of Graphics. Version 2. 1 pp. 1-189).

Woodworth, R. S. (1938). Experimental psychology. New York: Holt.

Woodworth, R. S., \& Schlosberg, H. (1954). Experimental psychology (Rev. ed.). New York: Holt.

Wundt, W. (1908). Grundzüge der physiologischen Psychologie [Principles of physiological psychology] (6th ed.) (6th ed., Vol. 1). Leipzig: Engelmann.

Piai, V, Roelofs, A, \& Van der Meij, R (2012). Event-related potentials and oscillatory brain responses associated with semantic and Stroop-like interference effects in overt naming. Brain Research, 1450, 87-101.

Piai, V, Roelofs, A, \& Schriefers, H (2011). Semantic interference in immediate and delayed naming and reading: Attention and task decisions. Journal of Memory and Language, 64, 404-423. 